\title{
Supervivencia global y supervivencia libre de progresión en pacientes con cáncer de ovario avanzado en la era del bevacizumab: experiencia en un centro oncológico de Medellín - Colombia.
}

Overall survival and progression-free survival in patients with advanced ovarian cancer in the bevacizumab era: Experience in an oncology center in Medellín - Colombia

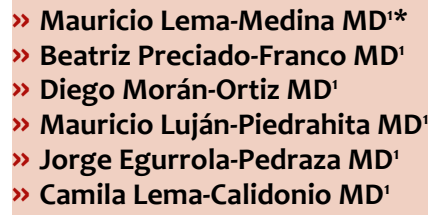

${ }^{1}$ Clínica de Oncología Astorga, Medellín - Colombia.

\section{Resumen}

Objetivo: Describir los aspectos clínicos, la supervivencia libre de progresión (SLP) y la supervivencia global (SG) en una cohorte de pacientes con cáncer de ovario avanzado y establecer las diferencias entre las tratadas con quimioterapia con o sin bevacizumab en la Clínica de Oncología Astorga, Medellín.

Materiales y métodos: Estudio observacional de una cohorte retrospectiva de pacientes que recibieron tratamiento entre 2012 y 2018, con análisis de supervivencia. Se realizó estadística descriptiva Kaplan-Meier para la supervivencia general y por grupo, y se estimó el cociente de riesgo (HR).

Resultados: Se incluyeron 49 pacientes. La edad mediana fue 63 años (RIQ: 54-68), 29 (59\%) con histología serosa, 24 (49\%) en estadio IV, 35 (71\%) requirieron cirugía, con citorreducción primaria en 23 ( $66 \%$ de las llevadas a cirugía). 24 (49\%) pacientes recibieron bevacizumab. La mediana de seguimiento fue de 24 meses, con 32 progresiones y 22 fallecimientos. Las medianas de SG en la cohorte total, con y sin bevacizumab fueron de 46 (IC95 \%: 16-75), 37 (IC95\%: 21-53) y 61 (IC95 \%: 15-106) meses, respectivamente. Las medianas de SLP en la cohorte total, con y sin bevacizumab fueron de 20 (IC95\%: 16-24), 18 (IC95\%: 13-23) y 36 (IC95\%: 13-59) meses, respectivamente. No se encontraron diferencias estadísticamente significativas entre los grupos para SG $(p=0.882)$ y SLP $(p=0.312)$.

\footnotetext{
* Autor para correspondencia: Mauricio Lema Medina. MD, Hemato oncólogo. Clínica de Oncología Astorga. Clínica SOMA. Correo electrónico: mauriciolema@yahoo.com Doi: https://doi.org/10.51643/22562915.77

Sociedad Colombiana de Hematología y Oncología. Este es un artículo Artículo en Acceso Abierto. Licencia Creative Commons Reconocimiento-NoComercial-SinObraDerivada (https://creativecommons.org/licenses/by-nc-nd/4.0/)
} 
Conclusiones: No se encontraron diferencias estadísticamente significativas, al comparar los grupos de pacientes que recibieron quimioterapia con y sin bevacizumab en esta cohorte de pacientes con cáncer de ovario avanzado.

Palabras clave: Neoplasias ováricas; bevacizumab; supervivencia libre de progresión; análisis de supervivencia.

\section{Abstract}

Objective: Describe the clinical characteristics, progression-free survival (PFS), and overall survival (OS) in a cohort of patients with advanced ovarian cancer and establish the differences between those treated with chemotherapy with or without bevacizumab at Clínica de Oncología Astorga, Medellín.

Material and methods: We carried out an observational study of a retrospective cohort of patients who received treatment between 2012 and 2018. OS and PFS of the treatment subgroups were compared using Kaplan-Meier curves and the log-rank test; also, the hazard ratio (HR) was estimated.

Results: We evaluated 49 patients. The median age was 63 years (IQR: 54-68). Among the patients evaluated 29 (59\%) had serous carcinoma, 24 (49\%) in stage IV, 35 (71\%) required surgery, primary cytoreductive surgery was performed in $23 / 35$ patients (66\%), and $24(49 \%)$ patients received bevacizumab. Median follow-up was 24 months, 32 patients developed disease progression, and 22 patients died. Median OS was 46 (95\% Cl 16-75), 37 (95\% Cl 21-53), and 61 ( $95 \% \mathrm{Cl} 15-106)$ months in the total cohort, with and without bevacizumab, respectively. Median PFS was 20 ( $95 \% \mathrm{Cl} 16-24), 18$ (95\% Cl 13-23), and 36 (95\% Cl 13-59) months in the total cohort, with and without bevacizumab, respectively. No statistically significant differences were observed in OS $(p=0.882)$ and PFS $(p=0.312)$ between the groups.

Conclusion: No statistically significant differences were observed when comparing groups of patients who received chemotherapy with and without bevacizumab in this cohort of patients with advanced ovarian cancer.

Keywords: Ovarian neoplasms; bevacizumab; progression-free survival; survival analysis

\section{Introducción}

El cáncer de ovario fue el más letal de los cánceres ginecológicos con 295.414 casos nuevos y 184.799 muertes por esta causa a nivel mundial, durante el 2018. En Colombia, el cáncer de ovario constituye la novena causa de muerte por cáncer en la mujer, con 2.414 casos nuevos por año y 1.252 muertes ${ }^{1}$.

El tratamiento del cáncer de ovario en estadios tempranos es quirúrgico, seguido por quimioterapia adyuvante en pacientes de alto riesgo ${ }^{2}$. Para efectos prácticos, las pacientes con carcinomatosis peritoneal primaria, carci- noma de trompa de Falopio y ovario estadios III y IV se manejan como una sola entidad, que a partir de este momento se denomina cáncer de ovario avanzado 3 . La supervivencia de las pacientes con carcinoma de ovario avanzado está fuertemente determinada por la calidad de la citorreducción quirúrgica obtenida al momento del diagnóstico ${ }^{4}$, seguida por tratamiento sistémico basado en platino más paclitaxel5. Para las pacientes que no son candidatas a citorreducción primaria, se puede obtener citorreducción de intervalo luego de quimioterapia con los mismos agentes ${ }^{6}$.

En el año 2011 se publicaron dos estudios so- 
bre la incorporación del Bevacizumab, un agente antiangiogénico, a la quimioterapia luego de cirugía en pacientes con alto riesgo de recaída ${ }^{7-9}$. En estos estudios se evidenció un incremento en la supervivencia libre progresión (SLP), y un posible incremento en la supervivencia global (SG) en pacientes con estadios más avanzados. Otros estudios han explorado el uso de bevacizumab en recaídas platino-sensibles ${ }^{10,11}$ o platino-resistentes ${ }^{12}$, también con incremento en la SG.

Existe una controversia aún no resuelta sobre el mejor momento para administrar el bevacizumab en pacientes con carcinoma de ovario. Para algunos no se ha establecido en forma concluyente el beneficio en la supervivencia global con su uso posterior a la cirugía, lo cual se ha convertido en razón suficiente para postergar su administración a otras líneas. Para otros, el incremento en la SLP ha sido motivo suficiente para su incorporación, aún en primera línea ${ }^{8}$. En el país, se dispone de escasa literatura que describa los desenlaces de supervivencia en pacientes con cáncer de ovario avanzado.

Este es un estudio que tiene como objetivo describir los aspectos clínicos, la supervivencia libre de progresión (SLP) y la supervivencia global (SG) de una cohorte de pacientes con diagnóstico de cáncer de ovario avanzado, y establecer las diferencias entre el grupo de pacientes que fueron tratadas con quimioterapia con o sin Bevacizumab en un centro oncológico en Medellín.

\section{Materiales y métodos}

\section{Diseño del estudio}

Estudio observacional de una cohorte retrospectiva de pacientes, con análisis de supervivencia, basado en "Real World Evidence" (RWE, por sus siglas en inglés, evidencia del mundo real).

\section{Participantes}

La población objetivo correspondió a pacientes con cáncer de ovario, carcinoma peritoneal primario y carcinoma de trompa de Falopio, en estadios avanzados (III, IV) o estadios tempranos (I, II) con recurrencia, que recibieron quimioterapia en la institución entre enero de 2012 y diciembre de 2018. No se incluyeron las pacientes con tumores borderline, de células germinales, sarcomas o metastásicos a ovario.

La estadificación se basó en criterios imagenológicos y en los reportes de cirugía y patología postoperatorios. Todas las pacientes recibieron el tratamiento estándar, el cual consiste en una combinación de platino con taxanos como primera quimioterapia sistémica.

Se definieron como sensibles al platino, aquellas pacientes que presentaron recurrencia luego de seis meses de terminado el esquema de quimioterapia que contenía una sal de platino.

\section{Variables}

Como variable independiente se definió la exposición a Bevacizumab (a criterio del médico tratante) independientemente del momento y el tiempo de la administración (neoadyuvancia/ adyuvancia/líneas siguientes de tratamiento). Las variables dependientes o desenlaces fueron la SG y SLP. La SG se definió como el tiempo en meses desde la fecha del diagnóstico por biopsia, hasta la fecha del último contacto o de muerte.

La SLP se definió como el tiempo en meses desde la fecha de inicio del primer tratamiento farmacológico, hasta la fecha de evidencia de progresión de la enfermedad o muerte. Las covariables fueron la edad, la escala ECOG, histología del tumor, estadio, tipo de cirugía citorreductiva y quimioterapia recibida.

\section{Fuente de los datos/mediciones}

La información de cada paciente se extrajo de la historia clínica electrónica de la institución (HiMedâ). Los datos fueron transferidos a una base de datos en Excelâ.

\section{Sesgos}

Se empleó un formulario de recolección de datos con todas las variables estandarizadas. Para controlar el sesgo de información, se verificaron los datos recolectados con los documentos fuente. Además, se llevaron a cabo los análisis de subgrupos por las covariables, para el control de la confusión. 


\section{Tamaño de muestra}

Se llevó a cabo un muestreo por conveniencia de todas las pacientes atendidas en la institución, se incluyeron en el análisis aquellas que cumplieron con los criterios de elegibilidad.

\section{Métodos estadísticos}

Las variables cualitativas se expresaron en frecuencias absolutas y relativas. Las variables cuantitativas se expresaron en medianas y rangos intercuartílicos (RIQ). Posteriormente, se compararon las diferencias entre las covariables descritas teniendo en cuenta la exposición a la variable independiente. Las diferencias en la distribución de las variables cualitativas con valores mayores a 5 se compararon con la prueba Chi-cuadrado de Pearson, mientras que para los valores esperados menores a 5, se utilizó la prueba exacta de Fisher. Las variables cuantitativas se compararon por la prueba $U$ de Mann-Whitney.

Para controlar el sesgo de información, las variables con más de un $20 \%$ de subregistro se excluyeron del análisis. En caso de tener menos de un $20 \%$ de subregistro, se realizó compensación por exclusión (cambio en $n$ de la muestra).

La supervivencia se estimó por el método del producto límite o de Kaplan-Meier, estratificado por la variable independiente. Las diferencias observadas gráficamente se evaluaron con la prueba de rangos logarítmicos (log-rank test). Posteriormente, se estimó el cociente de riesgo (HR). Para todas las estimaciones se estableció un alfa de 0.05 y un intervalo del $95 \%$ de confianza (IC95\%). Todos los análisis se realizaron en el programa estadístico SPSS versión 22.

\section{Aspectos Éticos}

El protocolo del estudio fue aprobado por un comité de ética independiente. No se requirió de consentimiento informado, al considerarse un estudio sin riesgo para las pacientes por ser un estudio retrospectivo, basado en revisión de historias clínicas. La confidencialidad fue garantizada con enmascaramiento de la identidad para todo el proceso de análisis.

El comité de ética autorizó el contacto telefónico con pacientes o familiares, para estable-

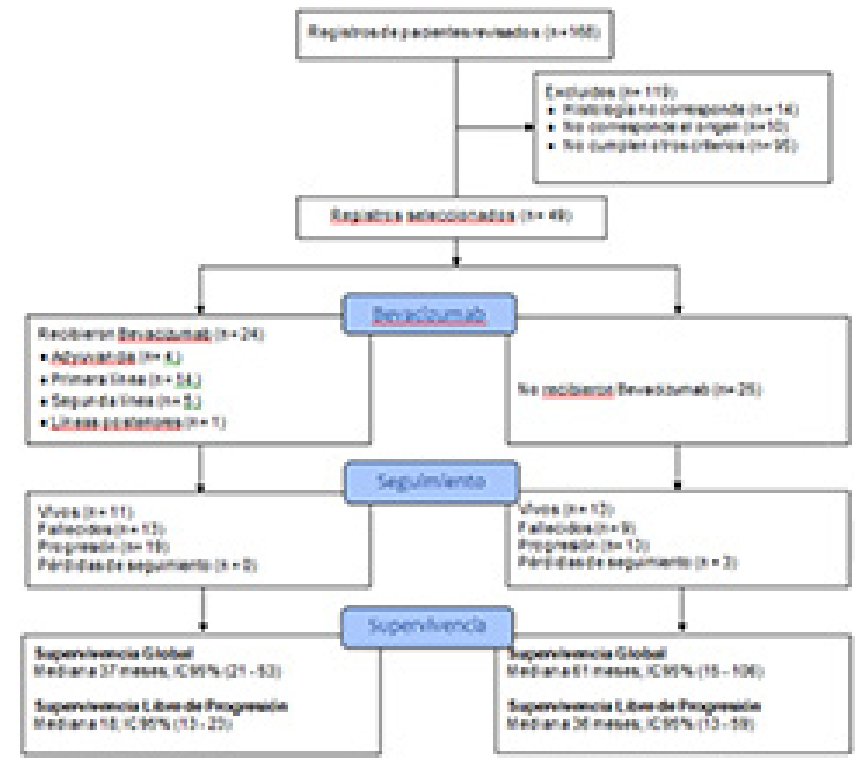

Figura 1. Flujograma de pacientes.

cer el estado vital en un grupo de pacientes perdidas de seguimiento.

\section{Resultados}

168 pacientes con diagnóstico de cáncer de ovario, peritoneal primario o de trompa de Falopio fueron atendidas en la institución entre los años 2012 y 2018. 49 cumplieron los criterios de elegibilidad. El cierre del seguimiento de las pacientes fue en junio 30 de 2019, con mediana de seguimiento de 24 meses (RIQ 12-41). No se obtuvo información del estado vital de tres pacientes (6.12\%) (Figura 1)

\section{Datos descriptivos}

La edad mediana de las pacientes fue 63 años (RIQ 54-68). El tipo de histología más frecuente del tumor fue seroso en 29 (59.18\%) seguido de adenocarcinoma en 11 (22.45\%). Al momento del diagnóstico 24 (49\%) pacientes se encontraron en estadio IV. Respecto a la clasificación del desempeño de las pacientes al momento del diagnóstico, se encontró un subregistro mayor al $20 \%$ de los datos por lo cual esta variable se excluyó del análisis. Fueron llevadas a cirugía 35 (71.43\%) pacientes. Se realizó citorreducción primaria a 23 de estas pacientes (65.71\%). Se realizó citorreducción completa en 9 (39.13\%) de ellas. A 12 (34.29\%) pacientes se realizó cirugía de intervalo, obteniéndose citorreducción 
completa en 10 (84\%) de ellas. Una paciente que recibió tratamiento en primera línea fue llevada a cirugía después de presentar respuesta y se incluyó en el grupo de cirugía de intervalo.

En cinco pacientes no se pudo establecer la extensión de la citorreducción practicada (cuatro con citorreducción primaria y una con cirugía de intervalo). En cuanto al primer esquema de quimioterapia recibido por las pacientes, 13 $(26.53 \%)$ recibieron quimioterapia neoadyuvante (11 pacientes con citorreducción de intervalo, una paciente falleció antes de la citorreducción y otra rechazó el procedimiento); 14 pacientes (28.57\%) recibieron quimioterapia adyuvante y 22 pacientes ( $44.89 \%$ ) iniciaron con quimiotera-

Tabla 1. Distribución de las características de las pacientes con cáncer de ovario avanzado de acuerdo a la administración de bevacizumab, Clínica de Oncología Astorga.

\begin{tabular}{|c|c|c|c|c|c|c|c|}
\hline & \multicolumn{2}{|c|}{$\begin{array}{l}\text { Total } \\
n=49\end{array}$} & \multicolumn{2}{|c|}{\begin{tabular}{|c|} 
Con \\
Bevacizumab \\
$n=24$
\end{tabular}} & \multicolumn{2}{|c|}{$\begin{array}{c}\text { Sin } \\
\text { Bevacizumab } \\
n=25\end{array}$} & $\rho^{*}$ \\
\hline \multicolumn{8}{|c|}{ Caracteristicas de las pacientes } \\
\hline Edad - mediana (RIQ) & 63 & $(54-69)$ & 65 & $(54-69)$ & 60 & $(54-69)$ & $0,688^{*}$ \\
\hline \multicolumn{7}{|l|}{ Histologia - n (\%) } & \multirow{6}{*}{$0.038^{*}$} \\
\hline Seroso & 29 & (59) & 18 & (75) & 11 & (44) & \\
\hline Adenocarcinoma & 11 & (23) & 4 & (17) & 7 & (28) & \\
\hline Endometroide & 2 & (4) & - & - & 2 & $(8)$ & \\
\hline Otros & 4 & (8) & $\cdot$ & - & 4 & (16) & \\
\hline Sin información & 3 & $(6)$ & 2 & $(8)$ & 1 & $(4)$ & \\
\hline \multicolumn{7}{|l|}{ Diagnóstico - n (\%) } & \multirow{3}{*}{$0.520^{\circ}$} \\
\hline Cáncer de ovario & 44 & $(90)$ & 22 & $(92)$ & 22 & $(88)$ & \\
\hline $\begin{array}{l}\text { Carcinoma peritoneal } \\
\text { Primario }\end{array}$ & 5 & $(10)$ & 2 & (8) & 3 & (12) & \\
\hline \multicolumn{7}{|l|}{ Estadio clinico - n (\%) } & \multirow{4}{*}{$0,616^{\circ}$} \\
\hline II & 2 & (4) & $\cdot$ & $\cdot$ & 2 & $(8)$ & \\
\hline III & 23 & (47) & 12 & $(50)$ & 11 & (44) & \\
\hline IV & 24 & (49) & 12 & $(50)$ & 12 & (48) & \\
\hline \multicolumn{8}{|c|}{ Tratamientos Recibidos } \\
\hline \multicolumn{7}{|c|}{ Cirugia - n (\%) } & \multirow{4}{*}{$0.581^{*}$} \\
\hline No establecido & 1 & (2) & $\cdot$ & - & 1 & (4) & \\
\hline No & 13 & (27) & 7 & (29) & 6 & (24) & \\
\hline Si & 35 & (71) & 17 & (71) & 18 & $(72)$ & \\
\hline $\begin{array}{l}\text { Citorreducción } \\
\text { Primaria }\end{array}$ & 23 & (66) & 12 & (71) & 11 & (61) & \multirow{8}{*}{$0,698^{3}$} \\
\hline Completa & 9 & (39) & 5 & $(42)$ & 4 & (36) & \\
\hline Incompleta & 10 & $(44)$ & 6 & $(50)$ & 4 & (36) & \\
\hline Sin Información & 4 & $(17)$ & 1 & $(8)$ & 3 & (28) & \\
\hline $\begin{array}{l}\text { Cirugia de } \\
\text { Intervalo }\end{array}$ & 12 & (34) & 5 & (29) & 7 & (39) & \\
\hline Completa & 10 & $(84)$ & 4 & $(80)$ & 6 & $(86)$ & \\
\hline Incompleta & 1 & $(8)$ & 1 & $(20)$ & - & - & \\
\hline Sin Información & 1 & (8) & - & $\cdot$ & 1 & (14) & \\
\hline \multicolumn{7}{|c|}{ Primer esquema de quimioterapia - $\mathrm{n}(\%)$} & \multirow{3}{*}{$0.147^{*}$} \\
\hline Neoadyuvancia - $\mathbf{n}(\%)$ & 13 & $(27)$ & 4 & $(17)$ & 9 & (36) & \\
\hline Adyuvancia - n (\%) & 14 & (28) & 6 & (25) & 8 & (32) & \\
\hline Primera linea $-\mathrm{n}(\%)$ & 22 & (45) & 14 & (58) & 8 & $(32)$ & \\
\hline \multicolumn{8}{|c|}{ Seguimiento } \\
\hline \multicolumn{7}{|c|}{ Progresión - n (\%) } & \multirow{3}{*}{$0,042^{\prime}$} \\
\hline $\mathrm{Si}$ & 32 & $(65)$ & 19 & $(79)$ & 13 & $(52)$ & \\
\hline No & 17 & (35) & 5 & (21) & 12 & (48) & \\
\hline \multicolumn{7}{|c|}{ Estado vital - n (\%) } & \multirow{4}{*}{$0,144^{*}$} \\
\hline Muerto & 22 & (45) & 13 & (54) & 9 & (36) & \\
\hline Vivo & 24 & (49) & 11 & (46) & 13 & (52) & \\
\hline Pérdida de seguimiento & 3 & (6) & $\cdot$ & $\cdot$ & 3 & (12) & \\
\hline
\end{tabular}

pia de primera línea.

En relación con el tratamiento, 33 pacientes recibieron una primera línea de tratamiento por enfermedad avanzada, para 22 pacientes fue el tratamiento inicial y para 11 pacientes fue por recaída. En cuanto a la sensibilidad al platino para primera línea esta fue del $54.54 \%$ (6/11) y para segunda línea del $39 \%$ (7/18). Recibieron tratamiento con bevacizumab 24 (49\%) pacientes, de estas el $50 \%$ lo recibieron en el primer esquema de tratamiento recibido (4 en adyuvancia, y 8 en primera línea) y el $50 \%$ más allá de la progresión.

Al comparar los grupos de pacientes que recibieron quimioterapia con o sin bevacizumab, no se observan diferencias estadísticamente significativas en cuanto a las características clínicas, supervivencia libre de progresión y supervivencia global. Los resultados se encuentran resumidos en la (Tabla 1)

\section{Supervivencia Global (SG)}

Al finalizar el seguimiento se presentaron 22 (44.9\%) muertes. La mediana de la SG para toda la población en estudio fue de 46 meses (IC95\%: 16-75) (Figura 2). Las pacientes que recibieron quimioterapia sin bevacizumab presentaron una mediana de la SG de 61 meses (IC95 \%: 15-106), mientras el grupo que recibió bevacizumab presentó una mediana de SG de 37 meses (IC95 \%: 21-53). Sin embargo, no hubo diferencias estadísticamente significativas en-

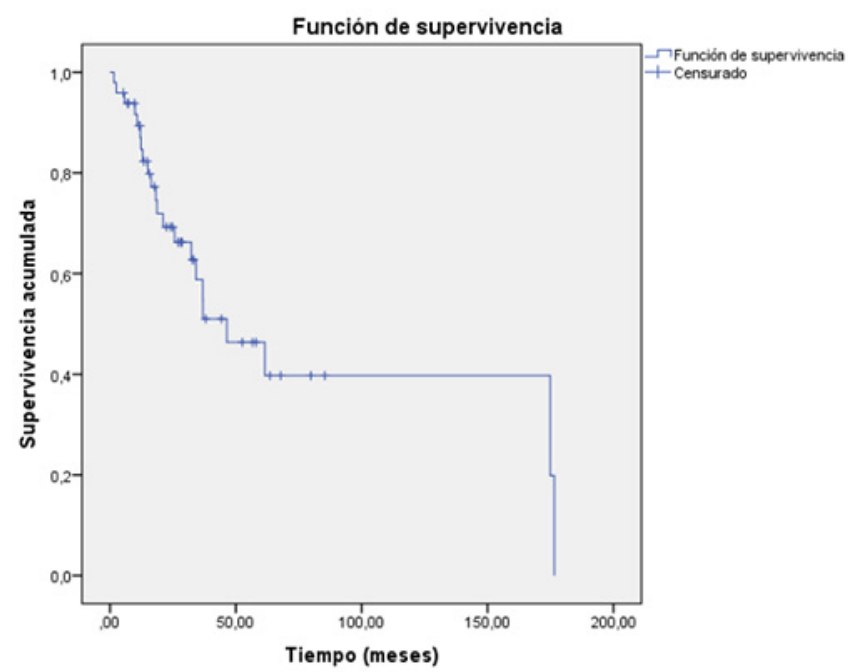

Figura 2. Curva de Kaplan-Meier para la supervivencia global en pacientes con cáncer de ovario avanzado. 
tre ambos grupos de tratamiento (log-rank test $=0.02 ; \mathrm{p}=0.882$ ). (Figura 3 ).

\section{Supervivencia Libre de Progresión (SLP)}

Durante el seguimiento se presentaron 32 casos de progresión de la enfermedad (65.31\%). La mediana de la SLP fue de 20 meses (IC95 \%: 1624) (Figura 4). De acuerdo a los grupos según la administración de bevacizumab, se obtuvo una mediana de SLP de 36 meses (IC95 $\%: 13-59)$ y de 18 meses (IC95 \%: 13-23) para las pacientes que no recibieron el esquema y las pacientes que sí lo recibieron, respectivamente. Sin embargo, no hubo diferencias estadísticamente significativas entre ambos grupos de tratamiento (log-rank test $=1.02 ; p=0.312)$ (Figura

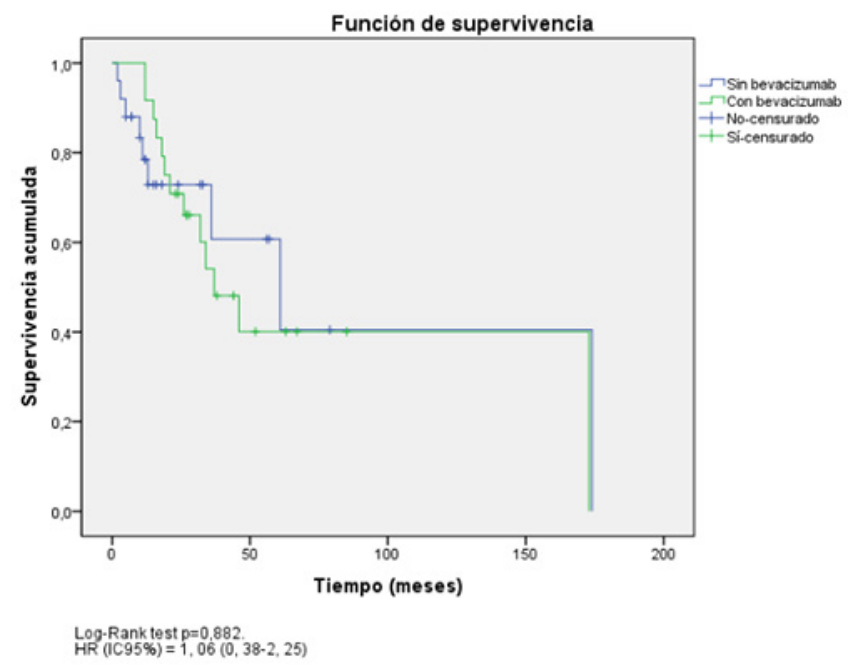

Figura 3. Curva de Kaplan-Meier para la supervivencia global en respuesta al bevacizumab en pacientes con cáncer de ovario avanzado.

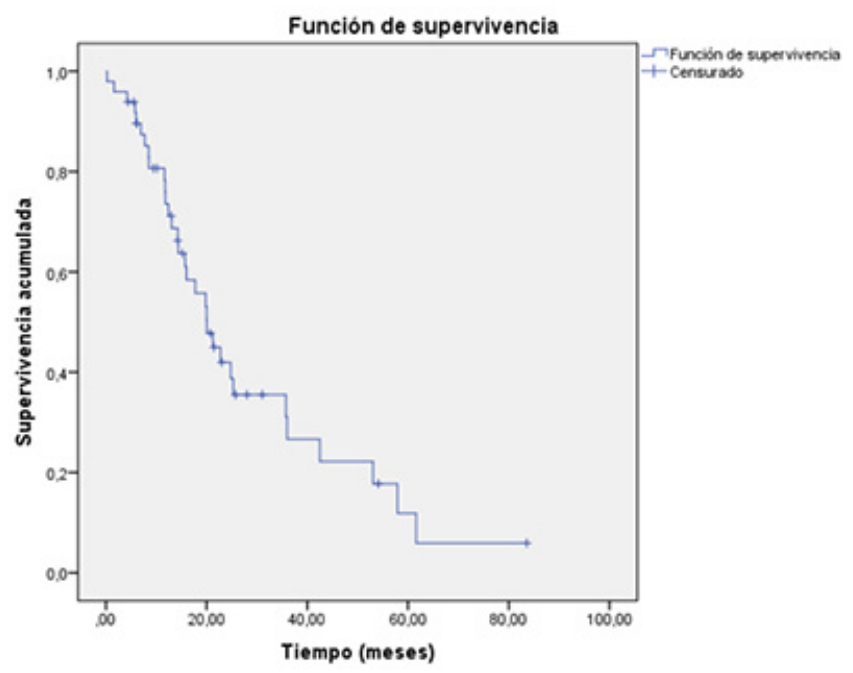

Figura 4. Curva de Kaplan-Meier para supervivencia libre de progresión en pacientes con cáncer de ovario avanzado.

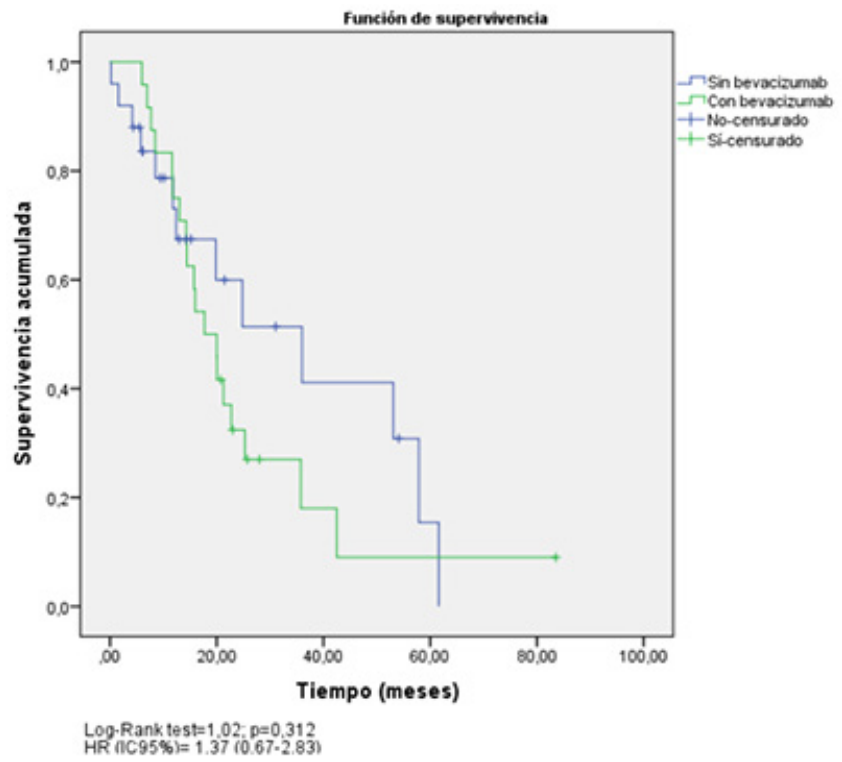

Figura 5. Curva de Kaplan-Meier para la supervivencia libre de progresión en respuesta al bevacizumab en pacientes con cáncer de ovario avanzado.

5).

A partir de las pacientes que recibieron neoadyuvancia, se comparó la SLP según el grupo de tratamiento. En el grupo de pacientes que no recibieron bevacizumab, se encontró que al final del seguimiento más del $50 \%$ de ellas no presentaron progresión (mediana no alcanzada). La mediana de la SLP para el grupo de pacientes que recibió bevacizumab fue de 12 meses (IC 95\%: 5-18). Sin embargo, no hubo diferencias estadísticamente significativas entre ambos grupos de tratamiento (log-rank test $=0.58 ; p=0.45)$.

\section{Cociente de riesgo}

No se evidenciaron diferencias estadísticamente significativas en el riesgo de muerte o progresión de la enfermedad entre los grupos estudiados (Figuras 3 y 5 ).

\section{Discusión}

Este es un estudio de RWE de quimioterapia con o sin bevacizumab en pacientes con cáncer de ovario avanzado en un centro oncológico de Medellín, Colombia.

El meta-análisis de Wu y colaboradores demuestra que el bevacizumab combinado con quimioterapia, mejora significativamente la SLP y SG en pacientes con alto riesgo de progresión 
y en pacientes con cáncer de ovario recurrente $^{13}$. La SLP con bevacizumab varía según el escenario clínico. En el estudio AURELIA en pacientes con resistencia a platino, la mediana de SLP fue de 6.7 meses $^{12}$.

En cambio, en el subgrupo de alto riesgo del ICON7 se observó una SLP de 15.6 meses luego de citorreducción primaria7. De igual forma la SG varía según el escenario clínico desde una mediana de 16.6 meses en el AURELIA a una de 39.3 meses en el grupo de alto riesgo del ICON7. En el presente estudio de RWE se incluyeron pacientes en diferentes momentos de la historia natural de la enfermedad, y se encontraron medianas de SLP y SG de 20 meses y 46 meses, respectivamente.

Se considera que los resultados de estas medidas de tiempo a evento, no muestran diferencias sustanciales con los reportados en la literatura.

\section{Conclusiones}

En el presente estudio no se logró mostrar un incremento en la SLP ni en la SG en el grupo de pacientes que recibió bevacizumab. Como posible explicación, se especula que ambos grupos de pacientes no son homogéneos si se considera la baja tasa de progresión del grupo no-bevacizumab que recibieron quimioterapia neoadyuvante (en donde el bevacizumab no está indicado).

A diferencia del grupo bevacizumab en el que se incluyeron pacientes que recibieron quimioterapia neoadyuvante, quienes sólo recibieron el biológico en algún momento después de la progresión (Tabla1).

Se reconocen las siguientes limitaciones de este estudio: tamaño muestral bajo, tiempo de seguimiento corto, naturaleza retrospectiva del estudio, ausencia de estandarización del uso del bevacizumab en la institución, pérdida de seguimiento de algunas pacientes, y la ausencia de información sobre aspectos como la citorreducción completa en aproximadamente el $10 \%$ de las pacientes, así como la escala de desempeño al inicio de tratamiento en $22 \%$ de las pacientes incluidas.

A pesar de las limitaciones descritas, se considera que este estudio aporta a la experiencia oncológica de pacientes con cáncer de ovario tratadas en Colombia, pues se describen desenlaces de supervivencia en el marco de la práctica usual del país. Los datos obtenidos no permiten concluir que el bevacizumab se asocia a prolongación de la supervivencia libre de progresión o de la supervivencia global de pacientes con cáncer de ovario avanzado incluidas en el estudio.

- Agradecimientos: Los autores agradecen al personal de la Unidad de Investigación de la Clínica de Oncología Astorga, por su colaboración en el desarrollo del estudio y la recolección de la información.

- Aspectos Éticos: El presente trabajo cumple con las normas de ética en investigación vigentes. De acuerdo con la Resolución 8430 de 1993, este estudio se clasifica en la categoría de investigación sin riesgo, dado que se empleó un método en el cual la información fue obtenida de fuentes secundarias y no se realizó ninguna intervención o modificación intencionada de las variables biológicas, fisiológicas, psicológicas o sociales de los individuos que participaron en el estudio, entre los que se consideran la revisión de historias clínicas. En ese orden, no fue necesario por parte de los investigadores la obtención del consentimiento informado de las pacientes de quienes se obtuvieron los datos.

- Fuente de Financiación: El estudio fue realizado con recursos de la Clínica de Oncología Astorga, Medellín.

- Conflictos de Interés: Los autores manifiestan no presentar ningún conflicto de interés 


\section{Referencias}

1. Bray F, Ferlay J, Soerjomataram I, Siegel RL, Torre LA, Jemal A. Global cancer statistics 2018: GLOBOCAN estimates of incidence and mortality worldwide for 36 cancers in 185 countries. CA Cancer J Clin [Internet]. 2018;68(6):394-424. https://doi.org/10.3322/caac.21492

2. NCCN Clinical Practice Guideline in Oncology. Ovarian Cancer Including Fallopian Tube Cancer and Primary Peritoneal Cancer. 2018. p. 1-126.

3. Prat J. Staging classification for cancer of the ovary, fallopian tube, and peritoneum. Int J Gynecol Obstet [Internet]. 2014 Jan 1;124(1):1-5. https://doi.org/10.1016/j.ijgo.2013.10.001

4. Griffiths CT. Surgical resection of tumor bulk in the primary treatment of ovarian carcinoma. Natl Cancer Inst Monogr. 1975; 42: 101-4. PMID: 1234624

5. McGuire WP, Hoskins WJ, Brady MF, Kucera PR, Partridge EE, Look KY, et al. Cyclophosphamide and Cisplatin Compared with Paclitaxel and Cisplatin in Patients with Stage III and Stage IV Ovarian Cancer. N Engl J Med [Internet]. 1996 Jan 4;334(1):1-6. https://doi.org/10.1056/ nejm199601043340101

6. Vergote I, Tropé CG, Amant F, Kristensen GB, Ehlen $\mathrm{T}$, Johnson $\mathrm{N}$, et al. Neoadjuvant Chemotherapy or Primary Surgery in Stage IIIC or IV Ovarian Cancer. N Engl J Med [Internet]. 2010 Sep 1;363(10):943-53. https://doi.org/10.1056/nejmoa0908806

7. Oza AM, Cook AD, Pfisterer J, Embleton A, Ledermann JA, Pujade-Lauraine E, et al. Standard chemotherapy with or without Bevacizumab for women with newly diagnosed ovarian cancer (ICON7): overall survival results of a phase 3 randomised trial. Lancet Oncol [Internet]. 2015 Aug 1;16(8):928-36. https://doi.org/10.1016/S14702045(15)00086-8

8. Tewari KS, Burger RA, Enserro D, Norquist BM, Swisher EM, Brady MF, et al. Final Overall Sur- vival of a Randomized Trial of Bevacizumab for Primary Treatment of Ovarian Cancer. Journal of Clinical Oncology [Internet]. 2019 Sep 37(26), 2317-2328. https://doi.org/10.1200/JCO.19.01009

9. Monk BJ, Huang HQ, Burger RA, Mannel RS, Homesley HD, Fowler J, et al. Patient reported outcomes of a randomized, placebo-controlled trial of Bevacizumab in the front-line treatment of ovarian cancer: A Gynecologic Oncology Group Study. Gynecol Oncol [Internet]. 2013 Mar 1;128(3):573-8. https://doi.org/10.1016/j.ygyno.2012.11.038

10. Aghajanian C, Blank $S \mathrm{~V}$, Goff BA, Judson PL, Teneriello MG, Husain A, etal. OCEANS: A Randomized, Double-Blind, Placebo-Controlled Phase III Trial of Chemotherapy With or Without Bevacizumab in Patients With Platinum-Sensitive Recurrent Epithelial Ovarian, Primary Peritoneal, or Fallopian Tube Cancer. J Clin Oncol [Internet]. 2012 Apr 23;30(17):2039-45. https://doi. org/10.1200/jco.2012.42.0505

11.Aghajanian C, Goff B, Nycum LR, Wang Y V, Husain $A$, Blank $S$ V. Final overall survival and safety analysis of OCEANS, a phase 3 trial of chemotherapy with or without Bevacizumab in patients with platinum-sensitive recurrent ovarian cancer. Gynecol Oncol [Internet]. 2015 Oct 1;139(1):10-6. https://doi.org/10.1016/j.ygyno.2015.08.004

12. Bamias A, Gibbs E, Khoon Lee C, Davies L, Dimopoulos M, Zagouri F, et al. Bevacizumab with or after chemotherapy for platinum-resistant recurrent ovarian cancer: exploratory analyses of the AURELIA trial. Ann Oncol [Internet]. 2017;28(8):1842-8. https://doi.org/10.1093/annonc/mdx228

13. Wu YS, Shui L, Shen D, Chen X. Bevacizumab combined with chemotherapy for ovarian cancer: an updated systematic review and meta-analysis of randomized controlled trials. Oncotarget [Internet]. 2017;8(6):10703-13. https:// doi.org/10.18632/oncotarget.12926 